\title{
ACIONAMENTO ELETRÔNICO DE MOTOR A RELUTÂNCIA: DETERMINAÇÃO DO PERFIL DA INDUTÂNCIA, CONTROLE DO CONJUGADO E COMUTAÇÃO SUAVE
}

\author{
L.P.B. de Oliveira, A.C. Oliveira, E.R.C. da Silva, A.M.N. Lima e C.B. Jacobina \\ Departamento de Engenharia Elétrica da Universidade Federal de Campina Grande \\ C.P. 10.105, Campina Grande, PB., 58.109-970 Brasil \\ Tel.: +55 (83) 310-1407, Fax: +55 (83) 310-1418, e-mail: edison@dee.ufcg.edu.br
}

\begin{abstract}
Resumo - Este artigo apresenta uma técnica para determinação da indutância em um motor a relutância. $O$ perfil da indutância é extraído, através de um algoritmo recursivo de Fourier, a partir da corrente de fase cuja amplitude é modulada devido à variação da indutância com a posição do eixo do motor quando o estator é alimentado por uma tensão de baixa freqüência. Em seguida, o artigo compara três técnicas utilizadas para reduzir a ondulação do conjugado do motor na região de baixa velocidade. Nessas técnicas, o perfil da indutância é simplificado durante o intervalo de comutação da corrente de uma fase para outra. Mostra-se que essas técnicas podem ser empregadas quando o motor de relutância é acionado por um conversor que opera com comutação suave. Resultados experimentais demonstram a viabilidade das soluções propostas.
\end{abstract}

Abstract - This paper first introduces a technique for determining the inductance of a switched reluctance machine. The inductance profile is extracted from the envelop of the phase current with the help of a recursive Fourier algorithm. The amplitude of the current is modulated due to the variation of the inductance with the shaft position (driven externally) when the motor is fed by an AC voltage. Next, the paper compares three simple control techniques used with the objective of reducing the torque ripple at low and medium speed of the motor. In these three techniques, the inductance profile is simplified during the interval of current commutation from one phase to another. It is shown that they can be used with a soft-switched version of the Miller's converter, of which the switches operate under simultaneous zero-voltage transition and zero-current transition (ZVZCT). Experimental results demonstrate the feasibility of the solutions proposed.

\section{INTRODUÇÃO}

A literatura técnica apresenta várias estruturas de conversores (inclusive estruturas a comutação suave), utilizadas no acionamento eletrônico de motores a relutância variável, SRM (do inglês "Switched Reluctance Motors") [1-6]. Entre os conversores a comutação suave, a versão com comutação suave do conversor de Miller [3], diferentemente do conversor de barramento CC [4], limita as tensões sobre seus componentes ao valor da tensão de alimentação. Ela, também, utiliza menos componentes do que outras versões, como a do conversor simétrico [5].

Dependendo do nível de potência, a utilização de diferentes estratégias de controle, para diferentes regiões do diagrama conjugado-velocidade, pode melhorar o desempenho global do SRM. Por exemplo, a eficiência é um critério importante de um modo geral, mas a ondulação do conjugado é o critério mais importante na região de baixa velocidade. Por isso, é imprescindível que a estrutura do controlador tenha flexibilidade suficiente para permitir a implementação dessas diferentes estratégias de controle [6].

Por outro lado, no SRM, enquanto o perfil da indutância da fase versus a posição do eixo, $L(\theta)$, determina os chamados ângulos de comutação, sua derivada em relação à posição, $d L(\theta) / d \theta$, define o projeto da forma de onda da corrente que resulta em um conjugado suave [7]. A técnica clássica para determinação do perfil da indutância de um SRM consiste em posicionar mecanicamente o eixo da máquina em uma determinada posição, enquanto que o enrolamento do estator é alimentado por um degrau de tensão. A corrente de fase é registrada e a indutância, naquela posição, é calculada a partir do tempo de subida da corrente do estator ou a partir da inclinação da curva fluxo vs corrente. A necessidade de posicionamento e travamento mecânico do eixo (a fim de evitar o alinhamento com a posição de equilíbrio quando a fase é energizada), além da utilização de um sistema servomotor, são os principais inconvenientes deste método.

Este trabalho propõe uma técnica que não requer o travamento do eixo da máquina. $\mathrm{O}$ valor da indutância é determinado a partir da envoltória da corrente, modulada em amplitude. Essa envoltória é extraída por meio de um algoritmo recursivo de Fourier [8]. Também, com a finalidade de reduzir a ondulação do conjugado em baixas velocidades, o trabalho investiga três estratégias para o controle da superposição de corrente durante o intervalo de sua transferência de uma fase para outra. As técnicas são empregadas em um inversor de Miller que opera simultaneamente com comutação a tensão nula e a corrente nula, ZVZCT (do inglês "zero-voltage-zero-current transition"). Sua flexibilidade permite a implementação de diferentes estratégias de controle. Resultados experimentais confirmam os estudos teóricos e demonstram a viabilidade das soluções propostas.

\section{O MOTOR A RELUTÂNCIA}

A representação de estado de um SRM é dada por

$$
\begin{aligned}
& \frac{d}{d t} \lambda_{k}\left(\theta, i_{k}\right)=v_{k}-r_{k} i_{k} \\
& \frac{d}{d t} \omega=-\frac{F}{J} \omega+\frac{1}{J}\left(T_{e}-T_{L}\right) \\
& \frac{d}{d t} \theta=\omega
\end{aligned}
$$

onde

é a posição angular (rad).

$v_{k}$ é a tensão aplicada ao $k$-ésimo enrolamento do estator (V), 
$i_{k}$ é a corrente no k-ésimo enrolamento do estator (A),

$\lambda_{k}(\theta, i)$ é o fluxo total no $k$-ésimo enrolamento $(\mathrm{Wb})$,

$r_{k}$ é a resistência do estator do $k$-ésimo enrolamento (Ohm)

$\omega$ é a velocidade angular ( $\mathrm{rad} / \mathrm{s})$,

$\theta$ é a posição angular (rad).

$T_{e}$ é o conjugado eletromagnético $(\mathrm{Nm})$

$T_{L}$ é o conjugado de carga $(\mathrm{Nm})$.

O conjugado eletromagnético produzido no SRM depende tanto da variação da indutância, função da posição angular $\theta$, como da corrente em cada enrolamento de fase (idênticas, exceto pela defasagem entre elas), ou seja,

$$
T_{e}=\frac{1}{2} \frac{d L_{k}(\theta)}{d \theta} i_{k}^{2}
$$

onde o subscrito $k$ da indutância e da corrente representa um dos subscritos dos enrolamentos $a, b$ ou $c$, etc.

Observa-se de (1) que o conversor deve fornecer apenas correntes unidirecionais. A estratégia mais simples consiste na energização de uma fase quando $d L_{k}(\theta) / d \theta$ se torna positiva e sua desenergização quando $d L_{k}(\theta) / d \theta$ se torna negativa. Os perfis idealizados das indutâncias do motor e das corrente nos enrolamentos são indicados nas Figs. 1(a) e 1(b). Na expansão da forma de onda da corrente $i_{a}$, podem ser consideradas três regiões, ou seja, a região de subida da corrente (SC), a região de topo da corrente (TC) e a região de descida da corrente (DC).

\section{A. Determinação do Perfil da Indutância}

Um perfil simplificado da indutância pode ser obtido considerando-se que a indutância varia linearmente entre seus valores máximo e mínimo. Tal perfil é apresentado esquematicamente na Fig. 2. Sua forma pode variar, dependendo se $\beta_{s}>\beta_{r}$ ou $\beta_{s} \leq \beta_{r}$ [11]. Da figura,

$$
\begin{aligned}
L_{a}(\theta) & =L_{\min } & & \text { for } \theta_{1} \leq \theta \leq 0 \\
& =L_{\min }+\theta & & \text { for } 0 \leq \theta \leq \beta_{s} \\
& =L_{\max } & & \text { for } \beta_{s} \leq \theta \leq \beta_{r} \\
& =L_{\max }-K\left(\theta-\beta_{r} \beta_{s}\right) & & \text { for } \beta_{r} \leq \theta \leq \beta_{r}+\beta_{s}
\end{aligned}
$$

em que $\beta_{s}$ e $\beta_{r}$ são parâmetros do SRM e $K=\left(L_{\max }-L_{\min }\right) / \beta$.

$\mathrm{Na}$ técnica apresentada a seguir, o perfil da indutância é obtido a partir da envoltória da corrente de fase, modulada em amplitude. A envoltória é extraída através de um algoritmo recursivo de Fourier [8].

\section{1) Envoltória da Corrente de Fase}

Se o eixo do motor é acionado externamente, a alimentação dos enrolamentos do estator de um SRM com uma tensão senoidal de amplitude e freqüência constantes gera uma modulação na corrente, devido à variação da indutância Assim, $L_{k}(\theta)$ pode ser determinada se a amplitude da componente fundamental desta corrente for conhecida.

$$
L_{k}(\theta)=\frac{\sqrt{\left[X_{L k}(\theta)\right]^{2}-r_{k}^{2}}}{2 \pi f_{c}}
$$

onde $f_{c}$ é a freqüência da tensão do estator e $I_{k}(\theta)$ a amplitude da componente fundamental da corrente do estator, dependente da posição instantânea do eixo. (a)

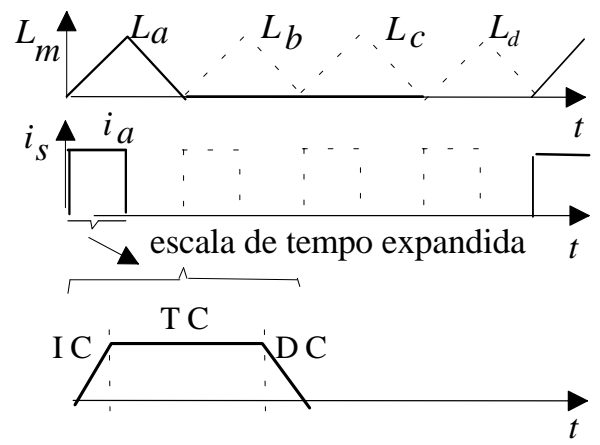

Fig. 1. Perfís ideais da indutância e da corrente.

\section{2) Algoritmo Recursivo de Fourier}

$\mathrm{O}$ algoritmo de Fourier de ciclo completo foi primeiramente proposto em [9] com a finalidade de determinar a amplitude e a fase a partir de suas medições uniformemente amostradas. $\left\{y(1), y(2), \cdots, y\left(N_{p}\right)\right\}$.

Para este algoritmo, supõe-se que as medidas podem ser representadas por

$y(l)=B \sin \left(\omega_{o} l \Delta \tau+\delta_{o}\right)=B_{c} \cos \left(\omega_{o} l \Delta \tau\right)+B_{s} \sin \left(\omega_{o} l \Delta \tau\right)$

onde $l=1,2, \ldots, N_{p}, \Delta \tau$ é o intervalo de amostragem, $N_{p}$ é o número de amostras no período, os valores de $B$ e $\delta_{o}$ sendo r

$$
B=\sqrt{B_{c}^{2}+B_{s}^{2}} \text { e } \delta_{o}=\arctan \left(\frac{B_{c}}{B_{s}}\right)
$$

onde $B_{c}=B \cos \left(\delta_{o}\right)$ e $B_{s}=B \sin \left(\delta_{o}\right)$.

A análise de Fourier de um dado sinal é uma extensão da equação (5), considerando-se um número maior de harmônicos. Para cada harmônico de ordem $m$, as amplitudes $B_{c}$ e $B_{s}$ são, então, determinados por [10]

$$
\begin{aligned}
& B_{c}(l)=B_{c}(l-1)+\frac{2}{N_{p}} y(l) \cos \left(\frac{2 \pi m l}{N_{p}}\right)-\frac{2}{N_{p}} y\left(l-N_{p}\right) \cos \left(\frac{2 \pi m l}{N_{p}}\right) \\
& B_{s}(l)=B_{s}(l-1)+\frac{2}{N_{p}} y(l) \sin \left(\frac{2 \pi m l}{N_{p}}\right)-\frac{2}{N_{p}} y\left(l-N_{p}\right) \sin \left(\frac{2 \pi m l}{N_{p}}\right)
\end{aligned}
$$

para uma janela deslizante com $N_{p}$ amostras, onde $l>N_{p}$, significando que foi coletado um ciclo completo de dados.

\section{ESTRATÉGIAS DE CONTROLE}

Já foi mostrado que, para a região de baixa velocidade de um SRM, é necessário se ter uma pequena ondulação de conjugado a fim de evitar oscilações de conjugado [6]. Essa condição pode ser obtida pela imposição de um perfil adequado para a corrente de fase. Entretanto, quando a velocidade aumenta, a importância da ondulação do conjugado diminui e a eficiência se torna o critério mais importante para o desempenho do sistema de acionamento.

No conversor trifásico de Miller (Fig. 3) [2] existem três modos básicos de operação para cada fase (ver Fig. 4). No Modo I (modo de potência) as chaves $S_{a}$ e $S_{f}$ conduzem de modo que $v_{a}=V_{d}$ (Fig. 4a); no Modo II (modo de circulação), Fig. 4(b), $S_{\mathrm{a}}$ está em condução (bloqueio) e $S_{f}$ em bloqueio (condução) fazendo com que a corrente circule através de $S_{a}\left(S_{f}\right)$ e $D_{f}\left(D_{a}\right)$ e acarretando $v_{a}=0$; no Modo III (modo de recuperação) as chaves $S_{a}$ e $S_{f}$ estão bloqueadas, e a corrente no enrolamento decresce através dos diodos $D_{a}$ e $D_{f}$ (Fig. $4 \mathrm{c}$ ), uma 
vez que $v_{a}=-V_{d}$

\section{A. Controle Individual da Corrente}

A forma de onda expandida da corrente $i_{a}$ da Fig. 1(b) permite a concepção de diferentes possibilidades de controle da corrente nas regiões SC, TC, e DC, através combinação dos Modos I, II e III, como discutido a seguir.

1) Região de Subida da corrente (SC). O Modo I $\left(v_{a}=V_{d}\right)$ pode ser usado para fazer crescer a corrente no enrolamento até que ela alcance o valor de topo; uma opção é imposição do perfil da corrente, empregando o Modo I com o Modo II (Estratégia $V_{d}, 0, V_{d}$ ) ou com o Modo III (Estratégia $V_{d},-V_{d}, V_{d}$ ).

2) Região do Topo de Corrente (TC). A corrente na região TC pode ser regulada ou pela Estratégia $V_{d},-V_{d}, V_{d}$, como indicado na Fig. 5(a), ou pela Estratégia $V_{d}, 0, V_{d}$, como indicado na Fig. 5(b); entretanto, pode apenas circular livremente [Estratégia 0, como indicado na Fig. 5(c)]; a Estratégia $O$ (ou, de pulso único) é a mais indicada para a região de alta velocidade, pois gera menos perdas do que as outras estratégias.

3) Região de Descida da Corrente (DC). A corrente pode ir para zero por aplicação da condição $v_{a}=-V_{d}$ ou por imposição de sua forma de onda.

\section{B. Controle da Corrente com Superposição}

Durante a transição entre duas fases, a ondulação do conjugado pode ser minimizada por uma técnica chamada de superposição. Nesse caso, as duas fases conduzem simultaneamente durante um período de tempo pré-determinado, uma delas na região $\mathrm{SC}$ e a outra na região DC. Os modos de operação, para a superposição de duas fases, são indicados na Fig. 6. A duração da superposição pode ser controlada pela utilização adequada desses modos.

A opção mais simples é a de se aplicar o Modo I $\left(v_{a}=V_{d}\right)$ para a fase que está entrando, enquanto o Modo II $\left(v_{a}=0\right)$ é utilizado para reduzir a corrente na fase que está saindo. Entretanto, durante a superposição, a indução de fase entrante é baixa e o uso do Modo I resulta em um crescimento rápido da corrente na fase. Como a indução da fase que está saindo é alta, o uso do Modo III acarreta uma descida longa da corrente, o que limita a freqüência de operação do sistema. Por isso, duas outras técnicas são discutidas a seguir.

1) Técnica I: Nesta técnica, a superposição de correntes é otimizada. A estratégia de controle do conjugado se baseia no seguimento de um contorno para cada fase do motor, de modo que a soma dos conjugados produzidos por cada fase seja constante e igual ao conjugado desejado, $T_{\text {ref }}$. Para isso, a função de contorno $f_{T}(\theta)$ é definida como

$$
T_{\text {total }}=T_{\text {ref }} f_{T}(\theta)
$$

onde $f_{T}(\theta)=\sum_{k=1}^{n} f_{k}(\theta)=1, f_{k}(\theta)$ sendo a função de contorno para a $k$-ésima fase. Considerando-se, cada função de contorno $f_{k}(\theta)$ como uma função de cosseno [9], o perfil da corrente individual de fase pode ser determinado a partir dos requisitos do conjugado e do perfil da indutância.

Uma simplificação é obtida ao se definir a função de contorno como um simples cosseno (controle cossenoidal):

$$
\begin{aligned}
& f_{a}=\frac{1}{2} \frac{d L_{a}}{d t} i_{a}^{2}=\frac{1}{2}\left[1-\cos \left(\theta-\theta_{o}\right)\right] \\
& f_{b}=\frac{1}{2} \frac{d L_{b}}{d t} i_{b}^{2}=\frac{1}{2}\left[1+\cos \left(\theta-\theta_{o}\right)\right]
\end{aligned}
$$

A escolha do ângulo de referência depende do perfil de indutância do motor utilizado. Entretanto, todas as indutâncias forem consideradas como lineares, durante o intervalo de transição, $\frac{d L_{a}}{d t}=K_{s}$ e as referências de correntes durante o intervalo de superposição, $\Delta t$, na transição da fase $a$ para a fase $b$ se tornam

$$
\begin{aligned}
& i_{a}^{*}=\sqrt{\frac{1}{K}\left[1-\cos \left(t-t_{o}\right)\right]} \\
& i_{b}^{*}=\sqrt{\frac{1}{K}\left[1+\cos \left(t-t_{o}\right)\right]} .
\end{aligned}
$$

Uma outra estratégia consiste em se considerar como linear, durante a transição, não só a indutância mas, também, o perfil da corrente em ambas as fases (controle em rampa), o que leva

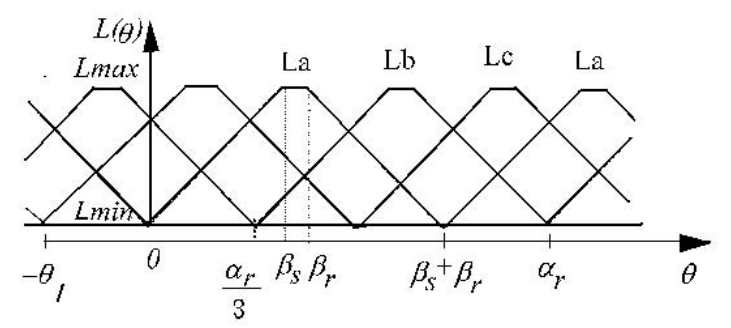

Fig. 2. Indutância variável no enrolamento de um SRM trifásico para $\beta_{r}>\beta_{s}$

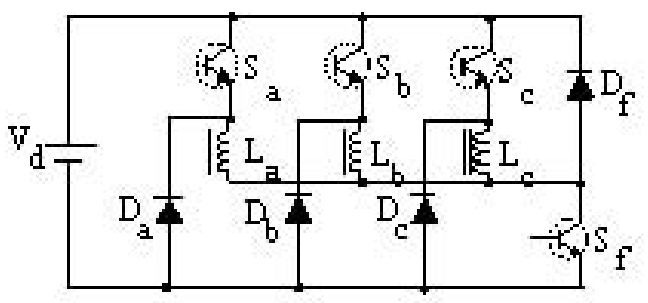

Fig. 3. Conversor trifásico de Miller.

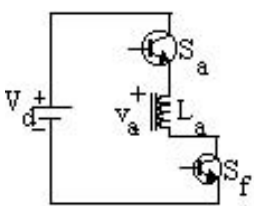

(a) Modo I

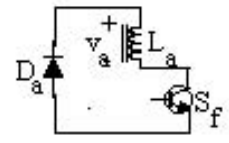

(b) Modo II

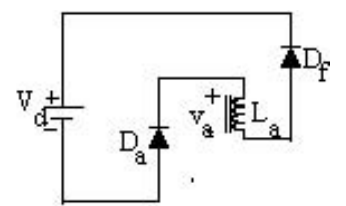

(c) Modo III
Fig. 4. Modos de operação para um braço de conversor.
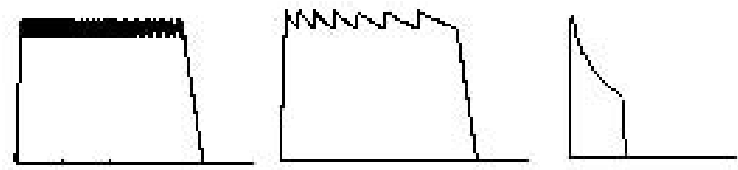

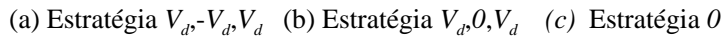


a referências mais simples, ou seja,

$$
\begin{aligned}
& i_{a}^{*}=\sqrt{\frac{2}{K_{s}}\left(1-\frac{t-t_{o}}{\Delta t}\right)} \\
& i_{b}^{*}=\sqrt{\frac{2}{K_{s} \Delta t}\left(t-t_{o}\right)} .
\end{aligned}
$$

2) Técnica II: Considerando-se lineares as indutâncias (1), durante a transição entre as fases $a$ e $b$, pode-se escrever:

$$
i_{a}^{2}+i_{b}^{2}=\frac{2 T_{r e f}}{K_{s}}
$$

Nesta técnica, aqui chamada de controle de subida, apenas a subida da corrente é controlada (fase $b$, no caso) enquanto a corrente na outra fase (fase $a$ ) é mantida em descida. Assim, a corrente de referência na fase $b$ torna-se

$$
i_{b}^{*}=\sqrt{\frac{2 T_{r e f}}{K_{s}}-i_{a}^{2}}
$$

Note-se que $\frac{2 T_{r e f}}{K_{s}}$ é o valor limite da corrente por fase. Um controle semelhante foi proposto em [12].

Os três tipos de controle foram simulados para as mesmas condições de operação. Os resultados da Fig. 7 indicam que o afundamento do conjugado, durante o intervalo de superposição, nos casos de uso dos controles cossenoidal e em rampa, é praticamente eliminado pelo uso do controle de subida. As ondulações nas formas de onda da corrente são resultado do controle das mesmas por histerese. Nos três casos, foi utilizada a Estratégia $V_{d}, 0, V_{d}$ o que permite menores perdas e uma ondulação menor com freqüência de chaveamento mais baixa do que com o uso da estratégia $V_{d},-V_{d}, V_{d}$. O controle de subida é uma técnica simples e não necessita nem de tabelas, nem de cálculo digital, nem da estimação ou medição precisa do ângulo de comutação.

\section{APLICAÇÃO À COMUTAÇÃO SUAVE}

A fim de testar a possibilidade de aplicação das estratégias de controle, discutidas acima, no controle de um SRM a comutação suave, foi escolhida uma versão do inversor de Miller. Diferentemente da configuração simétrica proposta em [5], a topologia apresentada na Fig. 8 pode operar com qualquer número de fases, apesar de ter um número menor de componentes. 0 capacitor $C_{r 2}$ e os diodos de $D_{a}$ a $D_{d}$ permitem que as chaves principais $S_{a}, S_{b}$ e $S_{c}$ operem com transição a tensão nula e a corrente nula, ZVZCT, simultaneamente. O sentido de conexão da chave $S_{r}$ permite que a chave $S_{m}$ opere com ZVZCT.

\section{A. Etapas na Mudança do Modo II para o Modo I}

A operação de mudança do Modo II para o Modo I, e vice-versa, no circuito da Fig. 8, pode ser descrita com o auxílio das oito etapas (ou sub-modos) relacionadas com os circuitos equivalentes seqüenciais indicados nas Figs. 9(a) to 9(i). Essas etapas são explicadas a seguir e relacionadas com as principais formas de onda apresentadas na Fig. 10.
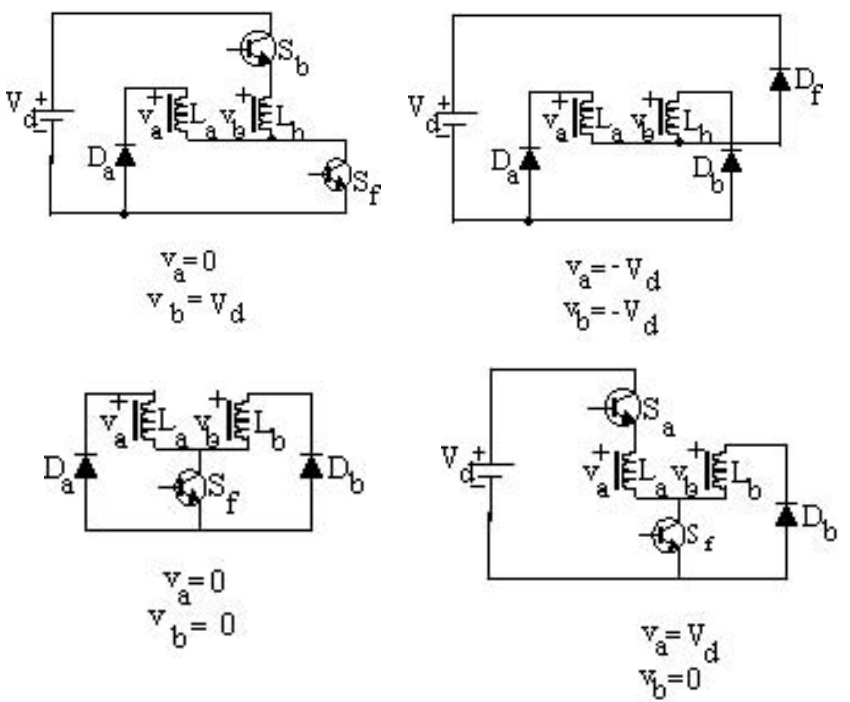

Fig. 6. Modos para superposição de duas correntes de fase.

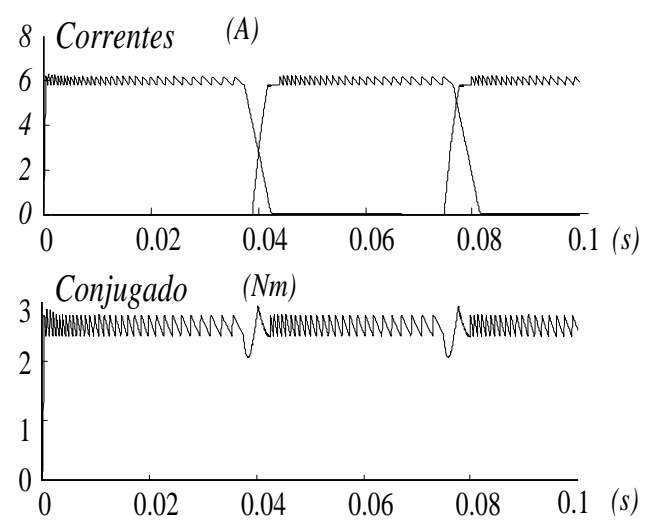

(a) Controle cossenoidal
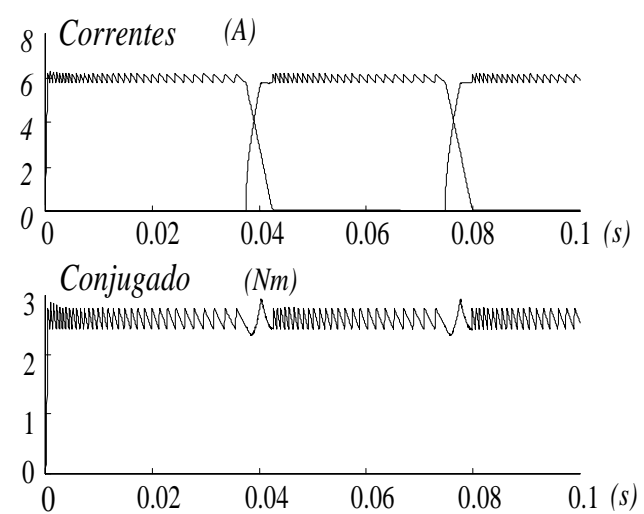

(b) Controle em rampa

Correntes (A)
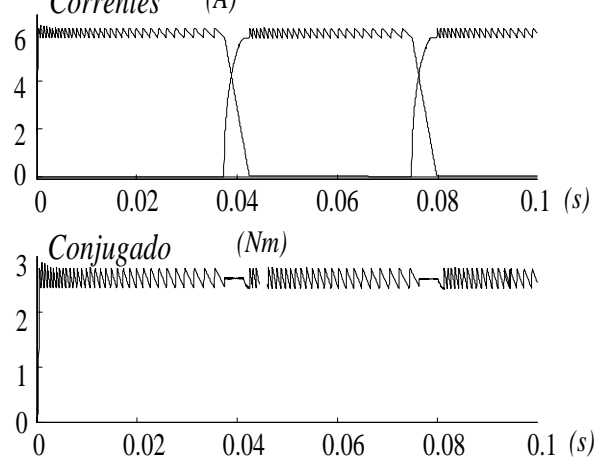

(c) Controle de subida

Fig. 7. Resultados de simulação das estratégias de controle. 


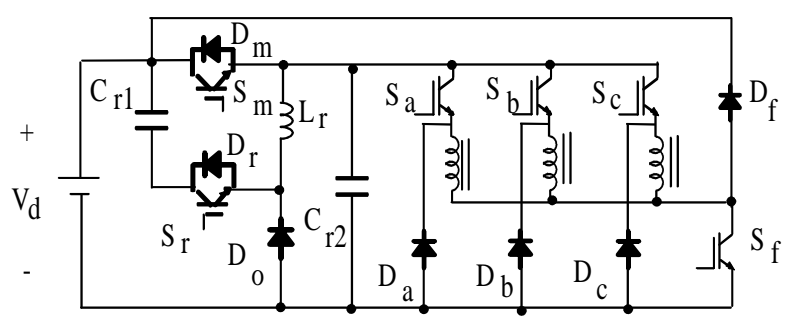

Fig. 8. Versão a comutação suave do convesor trifásico de Miller.

1) Etapa I (sub-modo de circulação, $t<t_{1}$ ): Suponha-se que, inicialmente, a corrente na fase $a, i_{a}$, esteja circulando através da chave $S_{f}$, do diodo $D_{a}$ e do enrolamento da fase $a, L_{a}$, como indicado na fig. 9(a). Uma simplificação é obtida considerando-se essa corrente como constante e igual $I_{a}$ durante o processo de comutação. Suponha-se, também, que o capacitor $C_{r 1}$ está carregado com uma tensão $V_{1}$, e que $C_{r 2}$ está completamente descarregado. As chaves $S_{m}, S_{r}$ e $S_{a}$ estão em estado de bloqueio, durante este intervalo.

2) Etapa II (primeiro sub-modo de ressonância, $t_{1}<t<t_{2}$ ): Nesta etapa, a s chaves $S_{r}$ e $S_{a}$ são disparadas com transições ZCT e ZVZCT, respectivamente, e a energia armazenada no capacitor $C_{r l}$ é transferida ressonantemente para o indutor $L_{r}$. Este intervalo termina quando a corrente ressonante atinge o valor $I_{a}$ da corrente $i_{a}$ e o diodo $D_{a}$ bloqueia. A condição necessária para que isso ocorra é

$$
2 V_{d} \sqrt{\frac{C_{r 1}}{L_{r}}}>I_{a}
$$

que a corrente atinja o valor $I_{a}$. no final do intervalo,

$$
v_{C r 1}\left(t_{2}\right)=V_{2}=V_{d}-\sqrt{4 V_{d}^{2}-\frac{L_{r}}{C_{r 1}} I_{a}^{2}}
$$

3) Etapa III (segundo sub-modo de ressonância, $t_{2}<t<t_{3}$ ): Após o bloqueio de $D_{a}$, ocorre uma nova ressonância, o capacitor $C_{r 2}$ sendo carregado com o auxílio da energia armazenada em $L_{r}$, no final da etapa anterior [Fig. 9(d)]. O diodo $D_{m}$ começa a conduzir quando a tensão em $C_{r 2}$ atinge o valor $V_{d}$. Para $\mathrm{C}_{\mathrm{r} 1} / \mathrm{C}_{\mathrm{r} 2} \gg>1$, a condição a ser observada é

$$
V_{d}-2 V_{2}>\frac{I_{a}}{\sqrt{k_{c}}}\left(\frac{\pi}{2}-\phi\right) \sqrt{\frac{C_{r 1}}{L_{r}}}
$$

onde:

$$
\begin{aligned}
& \tan \phi=\frac{V_{d}-V_{2}}{I_{a}} \sqrt{\left(k_{c}+1\right) \frac{C_{r 1}}{L_{r}}}, \quad k_{c}=\mathrm{C}_{\mathrm{r} 1} / \mathrm{C}_{\mathrm{r} 2} \quad \mathrm{e} \\
& V_{2}=V_{d}-\sqrt{4 V_{d}^{2}-\frac{L_{r}}{C_{r 1}} I_{a}^{2}} .
\end{aligned}
$$

4) Etapa IV (terceiro sub-modo ressonante, $t_{2}<t<t_{3}$ ): $C_{r 1}$ carrega ressonantemente até atingir o valor da fonte de tensão, quando $D_{m}$ começar a conduzir.

5) Etapa $V$ (sub-modo linear, $t_{4}<t<t_{5}$ ): Durante este intervalo, a corrente flui através de $D_{m}$ ou $S_{m}$. Inicialmente, o excesso de energia armazenada no indutor retorna para a fonte $V_{d}$ através do diodo $D_{m}$. A fim de garantir uma operação ZVZCT, um sinal é aplicado na base de $S_{m}$ durante a condução de $D_{m}$. Após seu bloqueio, a corrente começa a crescer em $S_{m}$ e a corrente em $L_{a}$ decresce.

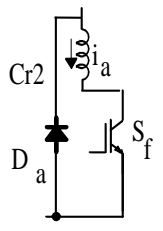

(a) Etapa I $\left(t_{1} \leq \mathrm{t}\right)$

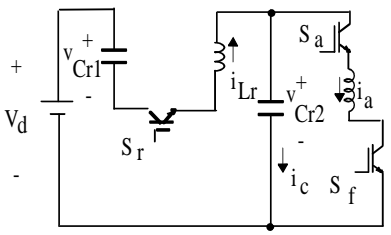

(c) Etapa III $\left(t_{2} \leq \mathrm{t} \leq t_{3}\right)$

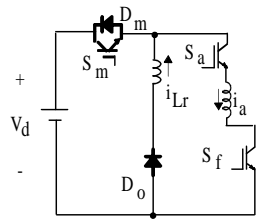

(e) Etapa VI $\left(t_{4} \leq \mathrm{t} \leq t_{5}\right)$

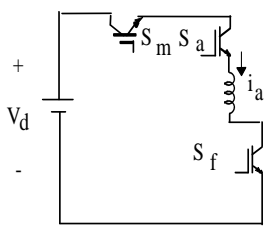

(h) Etapa VII $\left(t_{6} \leq \mathrm{t} \leq t_{7}\right)$

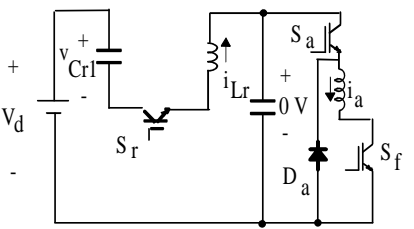

(b) Etapa II $\left(t_{1} \leq \mathrm{t} \leq t_{2}\right)$

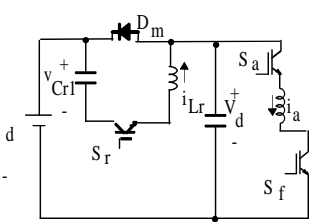

(d) Etapa IV $\left(t_{3} \leq \mathrm{t} \leq t_{4}\right)$

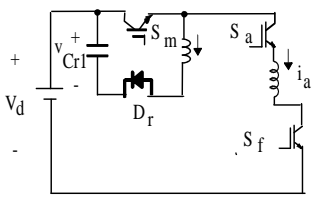

(f) Etapa VI $\left(t_{5} \leq \mathrm{t} \leq t_{6}\right)$

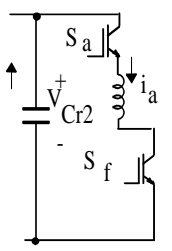

(i) Etapa VIII
Fig. 9. Circuitos equivalentes em um ciclo de operação.

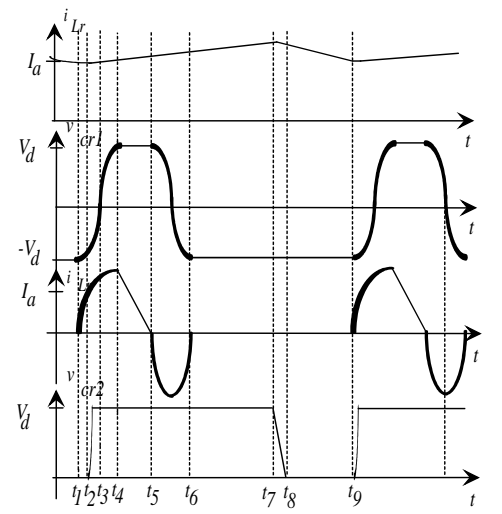

Fig. 10. Principais formas de onda para o circuito da Fig. 8.

6) Etapa VI (quarto sub-modo ressonante, $t_{5}<t<t_{6}$ ): Após a corrente em $S_{m}$ atingir o valor $I_{a}$, inicia-se uma nova oscilação, através do caminho $C_{r 1}-L_{r}-D_{a}-S_{m}$. A tensão no capacitor reverte e $V_{1}=-V_{d}$, no final da etapa.

7) Etapa VII (sub-modo de potência, $t_{6}<t<t_{7}$ ): Esta etapa durA até que o controle comande o bloqueio de $S_{m}$.

8) Etapa VIII (descarga de $C_{r 2}, t_{7}<t<t_{8}$ ): Após o bloqueio de $S_{m}$ com ZVT, a corrente no enrolamento $a, I_{a}$, descarrega $C_{r 2}$ de modo linear. Quando $v_{C r 2}$ atinge zero, $D_{a}$ começa a conduzir, 
encerrando o ciclo de operação.

Sequências semelhantes estabelecem a transição entre os Modos I e II e III e I. Isto garante a implementação de qualquer das técnicas de controle de corrente.

\section{RESULTADOS EXPERIMENTAIS}

A técnica proposta para determinação da indutância do SRM foi implementada através da plataforma experimental indicada na Fig. 11. A tensão de saída do amplificador de potência "push-pull", representado pelo Bloco A, alimenta o enrolamento da fase selecionada manualmente com o auxílio da chave $\mathrm{CH}$. Para cada fase, a forma de onda da corrente foi gravada para uma volta completa do eixo do motor, a fim de detectar possíveis assimetrias no perfil da indutância. A tensão da fase excitada, e as tensões induzidas nas outras fases, foram medidas através dos transdutores LV25-P (Blocos V). A corrente de fase foi medida através do transdutor LA25-NP (Bloco I). O sinal senoidal com freqüência de $100 \mathrm{~Hz}$ e amplitude de $3 \mathrm{~V}$ foi obtido do gerador de sinais $V_{i}$. Todos os sinais de tensão e corrente medidos foram digitalizados por conversores análogico/ digital de 12-bits de uma placa de aquisição instalada no PC.

Para os testes foi utilizado um SRM trifásico 12/8, acoplado mecanicamente a um encoder de 9-bits e a um motor CC, que impôs a velocidade de teste em $12 \mathrm{rpm}$. Esta velocidade deve ser baixa para que a posição do eixo permaneça essencialmente constante durante um ciclo da tensão senoidal de alimentação. A variação de 0.72 graus para o ciclo de $10 \mathrm{~ms}$, o que foi considerado satisfatório. A Fig. 12 mostra claramente a modulação de amplitude da corrente de fase devido à variação da indutância. Note-se que o valor médio da corrente de fase é nulo, o motor não desenvolvendo nenhum conjugado eletromagnético. Assim, pode-se girar facilmente o eixo, inclusive manualmente.

As envoltórias das formas de onda da tensão e da corrente foram determinadas pelo uso do algoritmo recursivo de Fourier. A figura 13 mostra os resultados obtidos para $N_{p}=250$ pontos e $\Delta \tau=40 \mu \mathrm{s}$. Como a amplitude da tensão aplicada ao estator é aproximadamente constante, a modulação observada nas correntes de fase é unicamente devida à variação da indutância.

A figura 14 mostra os perfis das indutâncias de fase do motor, que concordam razoavelmente com aqueles obtidos

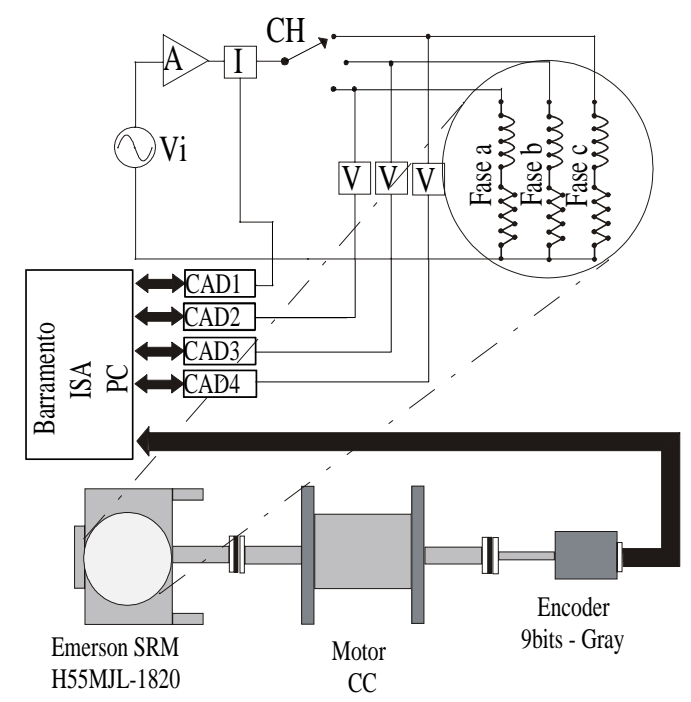

Fig. 11. Plataforma utilizada na determinação do perfil da

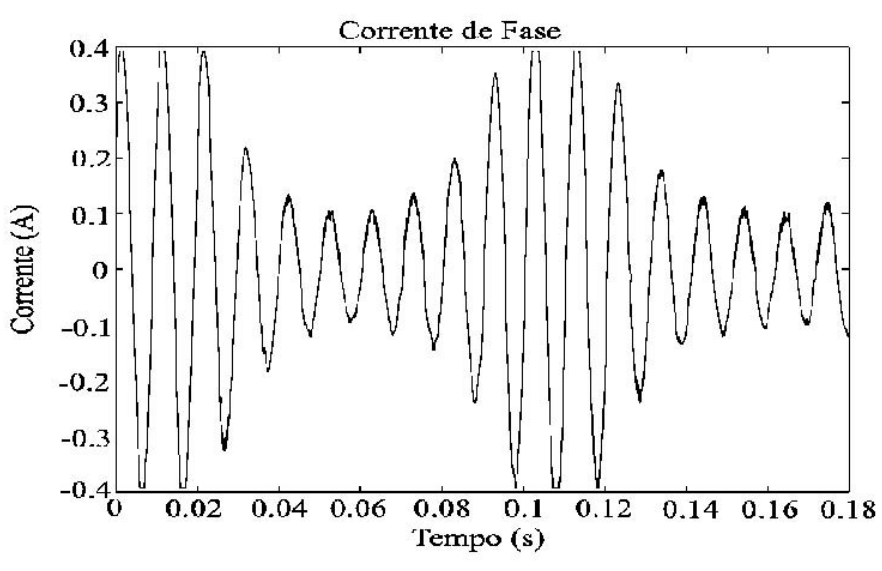

Fig. 12. Corrente de fase do SRM.

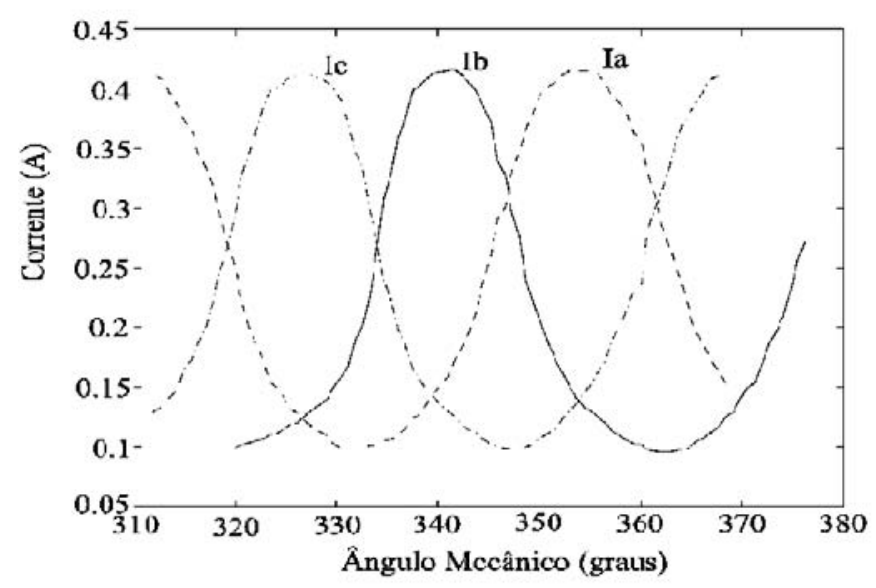

Fig. 13. Envoltória das correntes de fase obtida com o algorítmo recursivo de Fourier.

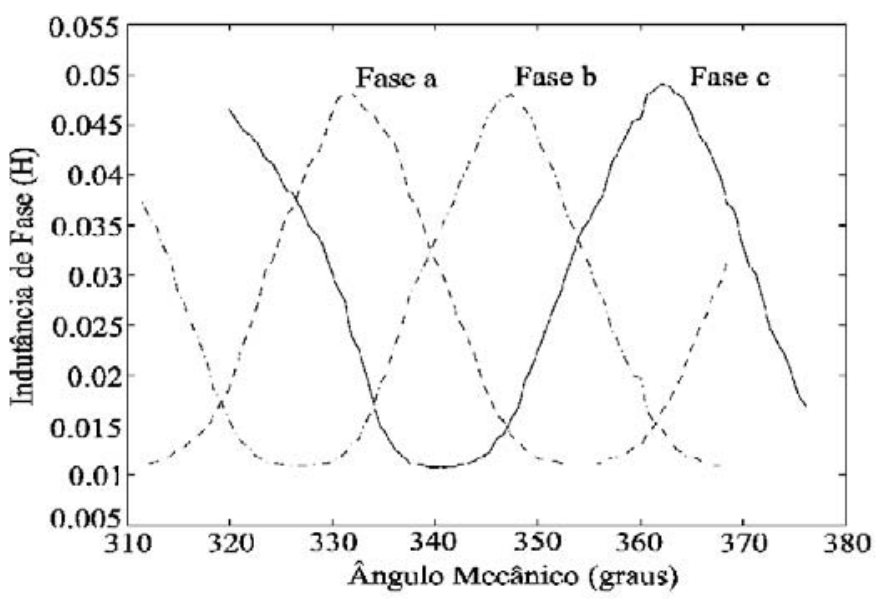

Fig. 14. Perfil da indutância de um motor 12/8, obtido com o algorítmo recursivo de Fourier.

através da técnica clássica, também implementada em laboratório. Outros resultados de testes, não mostrados, revelaram uma limitação da técnica com respeito à máxima freqüência da tensão de alimentação. Severas distorções nas corrente de fase aparecem quando a frequiência é aumentada. Isto pode ser atribuído à resposta em freqüência do material ferromagnético utilizado na construção do motor.

A verificação de aplicação dos princípios de controle da corrente, aplicados ao SRM a comutação suave da Fig. 8, também foi efetuada experimentalmente. Os valores utilizados para os parâmetros do conversor foram $V_{d}=50 \mathrm{~V}, C_{r l}=330 \mathrm{nF}$, $C_{r 2}=47 n F, L_{r}=2 \mu \mathrm{H}$. O SRM $12 / 8$ possui uma corrente 
nominal de $2.5 \mathrm{~A}, 120 \mathrm{VDC}$. Dos resultados da Fig. 14, os valores máximo $(49 \mathrm{mH})$ e mínimo $(11 \mathrm{mH})$ da indutância permitiram definir o perfil linear da indutância e o valor de $K_{s}$, durante o intervalo de superposição. Os resultados da Fig. 15, para uma velocidade de $477 \mathrm{rpm}$, correspondem aos casos de superposição com (1) controle cossenoidal, (2) controle linear de ambas as correntes e (3) controle da subida da corrente. A tensões nos terminais dos capacitores (ver Fig. 15c) se comportaram como esperado, com valores máximos iguais ao da fonte de alimentação. A ondulação do conjugado é menor no caso do controle da subida da corrente, o que confirma os resultados de simulação. $O$ conjugado foi estimado numericamente a partir dos resultados experimentais da corrente e do perfil da indutância utilizando a expressão (1). Outros resultados, não mostrados, indicam a flexibilidade do inversor investigado e sua operação em ZVZCT. Uma desvantagem da topologia investigada é a existência de uma chave no barramento CC. Também, valores baixos de corrente podem prejudicar a descarga de $C_{r 2}$. Entretanto, o circuito auxiliar pode ser modificado de maneira a retirar a chave do barramento $\mathrm{CC}$ e reduzir a dependência de descarga de $C_{r 2}$ com relação à corrente de carga. Na operação em velocidades elevadas a ondulação do conjugado perde importância face às perdas. Nesses casos pode ser utilizada a técnica 0 sem superposição, reduzindo as limitações de funcionamento.

\section{CONCLUSÕES}

Este trabalho mostra, inicialmente, como estimar o perfil da indutância de um motor a relutância chaveado eletronicamente, através de uma análise recursiva de Fourier da forma de onda da corrente de fase quando o estator é alimentado por uma fonte de tensão senoidal. A técnica não exige travamento do rotor em posições específicas, nem cálculo da inclinação da característica fluxo vs corrente. Testes experimentais com um motor 12/8 alimentado a $100 \mathrm{~Hz}$ mostraram que não é necessário um controle preciso da velocidade do eixo e que essa velocidade pode variar durante o teste. Entretanto, uma exigência é que a velocidade seja suficientemente baixa para que a posição do eixo permaneça essencialmente constante durante um ciclo da

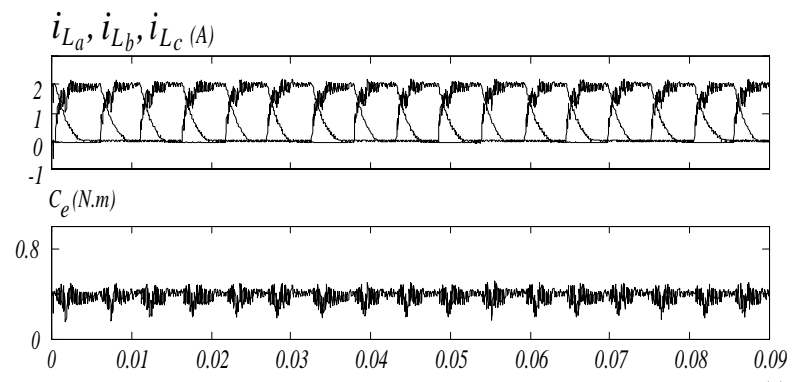

(a) Técnica I com controle cossenoidal.

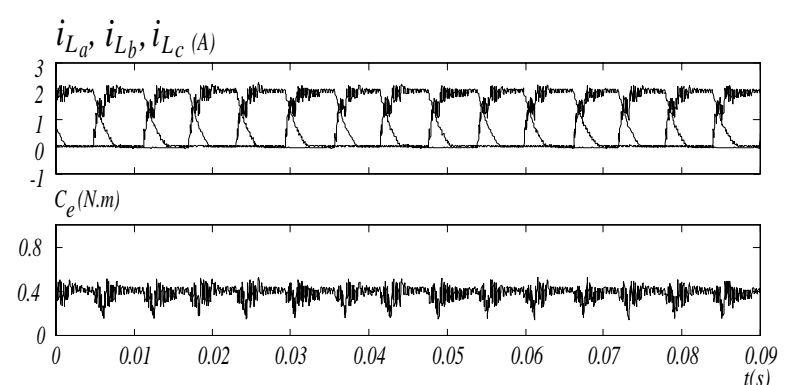

(b) Superposição: Técnica I com controle em rampa

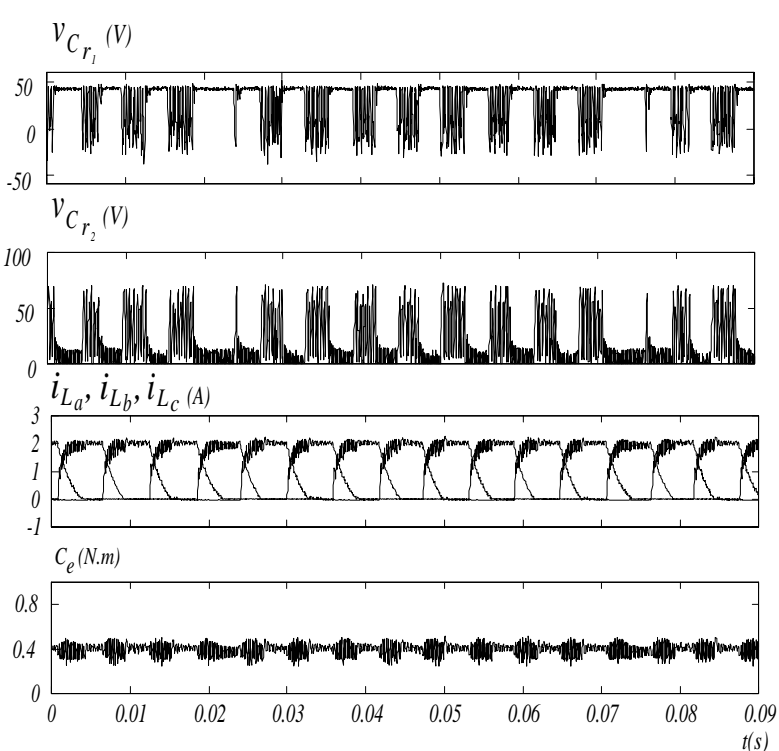

(c) Superposição: Técnica II (controle de subida).

Fig. 15. Resultados experimentais obtidos com o uso da Técnica $V_{d}, 0, V_{d}$ para regulação da corrente de topo.

tensão senoidal de alimentação. $O$ trabalho também comparou três técnicas de controle da forma de onda da corrente durante o intervalo de superposição, quando de sua transição de uma fase para outra. $\mathrm{O}$ testes experimentais mostraram que se obtém os melhores resultados, em relação à ondulação do conjugado, quando se controla apenas a subida da corrente em uma das fases durante o intervalo de superposição. Os resultados relativos ao conjugado foram obtidos numericamente "on line" a partir dos resultados experimentais da corrente e do perfil da indutância. Os testes, realizados com uma versão a comutação suave do conversor de Miller, comprovaram, ainda, a flexibilidade dessa topologia com relação à otimização do desempenho do sistema, através do uso de diferentes técnicas de controle.

\section{AGRADECIMENTOS}

Os autores agradecem à CAPES e CNPq pelo suporte financeiro, e à Emerson Motors, pelo fornecimento do motor utilizado nos testes experimentais.

\section{REFERÊNCIAS BIBLIOGRÁFICAS}

[1] A. Hava, V. Blasko, and T.A. Lipo, "A modified C-dump Converter for Switched Reluctance Machines", IEEE IAS'91 Conference Record, 1991, pp. 886-891.

[2] S. Vukosavic and V. Stefanovic, "SRM Inverter Topologies: a Comparative Evaluation", IEEE Trans. on Ind. Appl., Vol. 27, No. 6, Nov./Dec. 1991, pp. 1034-1047.

[3] E.R.C. da Silva, L.P.B. de Oliveira, A.M.N. Lima, and C.B. Jacobina, "A ZVZCT Power Converter Topology for Variable Reluctance Machine Drives", Proceedings of Brazilian Conference on Power Electronics (COBEP), 2001, pp. 804-809.

[4] E.R.C. da Silva, L.P.B. de Oliveira, C.B. Jacobina, and A.M.N. Lima, "New Soft-switched Power Converter Topologies for Variable Reluctance Machine Drives", IEEE 
PESC'99 Conference Record, pp. 826-831.

[5] G.H. Rim, W.H. Kim, and J.G. Cho, "ZVT Single Pulse-current Converter for Switched Reluctance Motor Drives", IEEE APEC'96 Conference Record, 1996, pp. 949-955.

[6] J. Reinert, R. Inderka, M. Menne, and R.W. De Doncker, "Optimizing Performance in Switched Reluctance Drives", IEEE APEC'98 Conference Record, 1998, pp. 765-770.

[7] I. Hussain and M. Ehsani, "Torque Ripple Minimization in Switched Reluctance Motor Drives by PWM Current Control", IEEE Trans. on Power Electronics, 2(1), 1986, pp. 83-88.

[8] L.A.L. Almeida, T.M. Oliveira, A.C. Oliveira, and C.B. Jacobina., "Algoritmo Robusto para Sincronização de Sistemas em Acionamento de Máquinas", Anaisdo XIII congresso Brasileiro de Automática (CBA), 2000, pp. 1509-1514.

[9] M. Ramamoorty, "Application for Digital Computers to Power System Protection", Journal of Inst. Electr. Eng. 52(10), 1972, pp. 235-238.

[10] A.C. Oliveira, C.B. Jacobina, and A.M.N. Lima, "Determining the Self-Inductance Profile of Switched Reluctance Motors using a Recursive Fourier Algorithm", Proceedings of Brazilian Conference on Power Electronics (COBEP), 2001, pp. 821-826.

[11] M.I. Valla and G. S. Buja, "Control Characteristics of the SRM Drives - part I: Operation in the Linear Region", IEEE Trans. on Ind. Electron., Vol. 38, N. 5, 1991, pp. 313-321.

[12] M.O. Bilgiç, V. Özbular, and A. Sabanovic, "Torque Riple Minimization of a Switched Reluctance Motor", IEEE APEC'95 Conference Record, pp. 406-410.

\section{DADOS BIOGRÁFICOS}

Levi Pedro Barbosa de Oliveira, nascido em 1967 em Maceió, Alagoas, é engenheiro eletricista (1994) e mestre (1997) em Engenharia Elétrica pela Universidade Federal da Paraíba e doutor (2001) pela Universidade Federal da Paraíba, Campina Grande. Desde 2001 é professor da Universidade Tiradentes, Aracaju. Suas áreas de interesse são: Eletrônica de Potência, Acionamento de Máquinas Elétricas, Automação Eletrônica de Processos Elétricos.

Alexandre Cunha Oliveira, nascido em 1970 em Fortaleza, Ceará, é engenheiro eletricista (1993) e mestre (1996) em Engenharia Elétrica pela Universidade Federal da Paraíba. Desde de 1996 é professor do Centro Federal de Ensino Tecnológico do Maranhão e atualmente encontra-se fazendo doutorado na Universidade Federal de Campina Grande, Campina Grande. Suas áreas de interesse compreendem acionamento de máquinas elétricas, conversores estáticos, fontes de alimentação chaveadas e instrumentação para medição de grandezas elétricas.
Edison Roberto Cabral da Silva, nasceu em Pelotas, RS, em 1942 e formou-se em Engenharia Elétrica pela Escola Politécnica de Pernambuco, em 1965. Obteve o título de Mestre pela COPPE da Universidade Federal do Rio de Janeiro e o de Doutor em Eng. Elétrica, pela Université Paul Sabatier de Toulouse, França, em 1968 e 1972, respectivamente. Foi docente da Universidade Federal da Paraíba desde 1967, estando atualmente na Universidade Federal de Campina Grande, onde é Professor Titular do Dep. Eng. Elétrica e Chefe do Laboratório de Eletrônica Industrial e Acionamento de Máquinas. Foi Professor Visitante na Universidade do Rio de Janeiro (1990) e na Universidade de Wisconsin-Madison, EUA (1990-1991). É, atualmente, presidente da Sociedade Brasileira de Automática, sendo sócio da SOBRAEP, e do IAS,PELS, IES e PES do IEEE. É membro da diretoria do IAS e seu representante para a América Latina. Suas áreas de interesse são conversores estáticos e acionamento de máquinas.

Antônio Marcus Nogueira Lima, nascido em 1958 em Recife, Pernambuco, é engenheiro eletricista (1982) e mestre (1985) em Engenharia Elétrica pela Universidade Federal da Paraíba e doutor (1989) pelo Institut National Polytechnique de Toulouse, Toulouse, France. De 1977 até 1982 foi professor da Escola Técnica Redentorista, Campina Grande. De 1982 até 1983 foi engenheiro projetista na Sul-América Philips, Recife. De 1983 até março de 2002 foi professor do Departamento de Engenharia Elétrica da Universidade Federal da Paraíba. Desde abril de 2002 é professor do Departamento de Engenharia Elétrica da Universidade Federal de Campina Grande. Suas áreas de interesse incluem Eletrônica de Potência, Acionamento de Máquinas Elétricas, Sistemas de Controle, Instrumentação Eletrônica e Identificação de Sistemas.

Cursino Brandão Jacobina, nascido em 1955 em Correntes, Pernambuco, é engenheiro eletricista (1978) e mestre (1980) em Engenharia Elétrica pela Universidade Federal da Paraíba e doutor (1983) pelo Institut National Polytechnique de ToulouseToulouse, France. De 1978 até março de 2002 foi professor do Departamento de Engenharia Elétrica da Universidade Federal da Paraíba. Desde abril de 2002 é professor do Departamento de Engenharia Elétrica da Universidade Federal de Campina Grande. Suas áreas de interesse são: Eletrônica de Potência, Acionamento de Máquinas Elétricas, Sistemas de Controle e Identificação de Sistemas. 